\title{
AVALIAÇÃO DE DESEMPENHO NO MINISTÉRIO DA CIÊNCIA E TECNOLOGIA: UM ESTUDO DO MODELO DE SINK E TUTTLE.
}

\author{
Wenner Glaucio Lopes Lucena \\ Doutor em Ciências Contábeis pela Universidade de Brasília - UnB \\ Professor do Programa Multiinstitucional e Inter-Regional de Pós-Graduação em Ciências Contábeis - \\ UnB/UFPB/UFRN \\ wdlucena@yahoo.com.br (Brasil)
}

\section{Gileno Fernandes Marcelino}

Pós-Doutor em Administração pela Universidade de São Paulo - USP

Prof. do Programa Multiinstitucional e Inter-Regional de Pós-Graduação em Ciências Contábeis UnB/UFPB/UFRN

gileno@marcelino.org.br (Brasil)

\section{RESUMO}

O objetivo principal deste artigo é verificar se o Modelo de Avaliação de Desempenho de Sink e Tuttle pode ser empregado no Ministério da Ciência e Tecnologia (MCT). A metodologia da pesquisa é do tipo descritiva e exploratória. O modelo de Sink e Tuttle possuem sete construtos, destes, foram escolhidas cinco, a saber: Eficácia, Eficiência, produtividade, Inovação e Qualidade. No total foram respondidos 142 (cento e quarenta e dois) questionários. O teste $\chi^{2}$ mostra no modelo de Sink e Tuttle que o grau de instrução influencia os níveis de produtividade nas Unidade de Pesquisa e Organização Social (UPOS). As unidades (INPE, CBPF, CETEM) são as que apresentam maior nível de tecnologia. $\mathrm{Na}$ análise da Correlação teve-se 8 variáveis que mais se destacaram e ficou evidente que o principal construto do modelo para as UPOS foi o da eficiência. No tocante às variáveis mais importantes, as duas principais foram eficiência no trabalho e qualidade, reforçando a idéia de que as características das unidades influenciam o desempenho das unidades. É consenso entre as unidades que a força de trabalho está aquém das necessidades de cada unidade.

Palavras-Chaves: Avaliação de Desempenho; Modelo Sink e Tuttle; MCT. 


\section{INTRODUÇÃO}

O tema avaliação de desempenho está enfrentando um crescente interesse nos âmbitos acadêmico e administrativo. Isso, na sua maior parte, é devido à ampliação dos modelos de performances exigidas pelo atual ambiente competitivo e à ruptura de paradigmas nas organizações Ghalayini \& Noble, (1996); Rolstadas, (1998); Taticchi \& Balachandran, (2008). Neste caso, o Ministério da Ciência e Tecnologia (MCT) é tratado como um órgão público representado pelas Unidades de Pesquisas e Organizações Sociais (UPOS).

As organizações rotineiramente avaliam o desempenho de indivíduos, atividades e subunidades. Por exemplo, as avaliações de desempenho frequentemente são usadas para alocar os recursos dentro da organização, decidir sobre as ações corretivas, definir metas de desempenho futuro, desenvolver ou aperfeiçoar as estratégias e identificar necessidades de treinamento e desenvolvimento. Além disso, a avaliação de desempenho é de fundamental importância nas organizações, e os dados financeiros e não financeiros servem como uma contribuição essencial na formação destas avaliações Foster \& Young, (1997); Ittner; Larcker, (2001).

$\mathrm{O}$ processo de gerenciamento dentro das organizações quase sempre é confrontado com o comportamento organizacional em que se encontra a instituição, extrair o máximo dos gestores e empregar um processo de melhoria contínua é inerente a qualquer organização, seja ela pública ou privada.

A despeito deste assunto, tem-se no Modelo de Avaliação de Desempenho de Sink e Tuttle os pressupostos para caracterizar este artigo. Assim, Da Paz (2003, pp. 2) enfatiza que "uma das maiores distorções que se observa no setor público, hoje, diz respeito aos critérios utilizados para avaliar o desempenho das suas entidades".

Desta forma, faz-se a seguinte indagação: Quais variáveis do Modelo de Avaliação de Desempenho de Sink e Tuttle podem ser aplicadas nas Unidades de Pesquisas e Organizações Sociais (UPOS) contribuindo para o seu alinhamento estratégico?

Assim, parte-se da premissa que os órgãos públicos realizam avaliação de desempenho com o objetivo de verificar resultados.

A outra premissa é que as (UPOS) possuem um alinhamento estratégico, logo elas estariam preocupadas em atender as exigências colocadas pelo Ministério da Ciência e Tecnologia via plano diretor, termo de compromisso e contrato de gestão, assim poderia se ter uma avaliação de desempenho. 
O objetivo principal deste artigo é verificar se o Modelo de Avaliação de Desempenho de Sink e Tuttle pode ser empregado nas Unidades de Pesquisas e Organizações Sociais e se ele contribui para um melhor alinhamento estratégico no Ministério da Ciência e Tecnologia.

\section{FUNDAMENTAÇÃO TEÓRICA}

\subsection{Modelo Sink e Tuttle}

Uma das principais abordagens clássicas para medição de desempenho é apresentada pelo modelo de Sink \& Tuttle (1993). As aplicações deste modelo de desempenho de um sistema organizacional referem-se a uma complexa inter-relação entre os seguintes sete critérios de desempenho: Eficácia, Eficiência, Qualidade, Produtividade, Qualidade no trabalho, Inovação e Lucratividade. Rolstadas, (1998).

O modelo de Sink e Tuttle defende que um sistema de medição de desempenho precisa estar integrado ao sistema de gestão da organização e voltado para a melhoria contínua. Para Schmidt, Santos \& Martins (2006) esse modelo é abrangente e integrado e apresenta sete critérios de desempenho, a partir dos quais um sistema organizacional deverá ser medido pela: eficácia, eficiência, qualidade, produtividade, qualidade de vida do trabalho, inovação e lucratividade.

Assim Sink \& Tuttle (1993) enfatizam que o foco deve ser, primeiro, sobre a eficácia, ou seja, quais são as coisas certas a fazer. Em seguida, deve-se começar a trabalhar a eficiência e qualidade. Se estes três conceitos estão em consonância, o resultado é muito provável que seja um processo produtivo para a organização. Qualidade de vida no trabalho e inovação são vistos como mediadores para a equação, pois eles podem aumentar e diminuir o desempenho. A manutenção de elevados desempenho nessas áreas, resultam a curto prazo muitas vezes na rentabilidade das organizações. Com isso, os resultados a longo prazo apresentam excelência, sobrevivência e crescimento.

Sink \& Tuttle (1993) vislumbram uma estrutura sistêmica da organização em que elencam cinco elementos: o sistema a jusante, entradas, processamentos, saídas, e o sistema a montante; de posse destes elementos, eles traçam seis indicadores de qualidade, um representando cada elemento e no final o sexto indicador monitora a qualidade do processo gerencial da organização.

Embora muita coisa fosse modificada nas organizações desde a introdução pela primeira vez do modelo de Sink e Tuttle, esses sete critérios de desempenho são ainda importantes. No entanto, o modelo tem várias limitações. Por exemplo, ele não considera a necessidade de flexibilidade nem leva em conta a perspectiva do cliente.

Revista de Administração e Inovação, São Paulo, v. 11, n.2, p. 51-70, abr./jun. 2014. 
Assim, o modelo de sete critérios de desempenho inicia-se com a visão da organização estabelecendo os objetivos específicos, passando por um processo de medição dos indicadores-chave, finalizando com o feedback da implementação da estratégia desejada Schmidt, Santos \& Martins (2006) Rolstadas (1998); Sink \& Tuttle, (1993). Todo este processo se torna possível pelo comprometimento dos funcionários e uma equipe de implementação que a todo o momento busca alinhar estrategicamente as necessidades da organização no tocante aos sete critérios propostos no modelo.

Sink \& Tuttle (1993) estruturam o sistema organizacional em sete critérios de desempenho que se dividem em cinco fases distintas, fazendo uma adaptação à realidade do setor público. Focando o Ministério da Ciência e Tecnologia, mais especificamente as Unidades de Pesquisas, pode-se dizer que os sistemas a montante tratam de cada unidade de pesquisa que por meio dos dados de entrada (Inputs) apresentam as informações que serão processadas de acordo com o planejamento estratégico. Os termos de contrato de gestão e os indicadores de avaliação de desempenho são os Outputs, com isso chega-se à resposta junto à Sociedade e à Subcoordenação das Unidades de Pesquisas (SCUP), no sentido de divulgar se essas unidades estão realmente desempenhando seu papel.

\subsection{Unidades de Pesquisas e Organizações Sociais}

Este trabalho visou analisar as Unidades de Pesquisas e Organizações Sociais pertencentes ao Ministério da Ciência e Tecnologia. Para isto, cabe nesta parte apresentar de forma sucinta as principais divisões, conceitos, organizações e classificações destas unidades, bem como suas estruturas e suas principais atividades.

De acordo com Marcelino, Matias-Pereira \& Berbert (2008) foi com a chegada da família imperial portuguesa que tiveram início de fato as pesquisas científicas no Brasil.

Durante o século XIX, existiu uma intensa "colonização" das pesquisas científicas, em que se teve a colaboração de cientistas europeus e americanos que contribuíram e ajudaram a criar as primeiras instruções de pesquisas no país. Marcelino, Matias-Pereira \& Berbert (2008).

Desta forma, as instituições de pesquisa avançaram e o período pós Segunda Guerra Mundial foi crucial para o surgimento de novas instituições científicas. Em 1951, foi criado o Conselho Nacional de Pesquisas - $\quad$ hoje Conselho Nacional de Desenvolvimento Científico e Tecnológico (CNPq) - e a Campanha Nacional de Aperfeiçoamento do Ensino Superior - atualmente Fundação Coordenação de Aperfeiçoamento de Pessoal de Nível Superior (CAPES) -, instituições fundamentais para o salto da ciência e da tecnologia no Brasil. Depois de mais cinco anos foram criadas a Comissão Nacional de Energia Nuclear (CNEN) e a 
Comissão Nacional de Atividades Espaciais (CNAE) - hoje em dia Agência Espacial Brasileira (AEB). No ano de 1966, surgiu a Financiadora de Estudos e Projetos (FINEP), para auxiliar as instituições de pesquisas e empresas tecnológicas. MDIC/CNI/SEBRAE, 2002 apud Marcelino, Matias-Pereira \& Berbert (2008).

Mesmo com todas essas instituições, o Ministério da Ciência e Tecnologia só veio a ser criado em 1985, para desempenhar a missão de aplicar políticas de desenvolvimento científico e tecnológico, bem como, de inovação. (MCT, (2009); Marcelino, Matias-Pereira \& Berbert (2008).

Com isso, o MCT passou por várias reestruturações, até chegar à estrutura atual: seu organograma contempla 5 (cinco) entidades vinculadas, 17 (dezessete) unidades de pesquisa que se subdividem em Unidade de Pesquisa Científica e de Pesquisa Tecnológica e de Inovação, 06 (seis) unidades subordinadas à CNEN e 03 (três) empresas públicas, discriminadas a seguir: (MCT, 2009).

Segundo Marcelino, Matias-Pereira \& Berbert (2008), em meados de 2000, o MCT sofreu uma reestruturação e apresentou essa nova configuração formada a partir das unidades de Pesquisas e as Organizações Sociais. Desta forma, o MCT passou a gerenciar e controlar essas unidades e organizações, apesar de se tratar de instituições diversificadas, com pensamentos e estruturas organizacionais diferentes. Marcelino, Matias-Pereira \& Berbert (2008).

Partindo do pressuposto que existia uma diversificação das unidades, o MCT verificou a necessidade de identificar a situação em que se encontravam as unidades de pesquisas e, em meados de 2000, foi constituída a “Comissão Tundisi” (recebeu esse nome por ser presidida pelo Prof. José Galizzia Tundisi, ex-presidente do CNPq). Depois de uma série de discussões com o MCT, a Sociedade Brasileira para o Progresso da Ciência (SBPC), a Academia Brasileira de Ciência (ABC), e com os próprios diretores das unidades, foi elaborado o "Relatório Tundisi". MCT/CGEE, (2002); Marcelino, Matias-Pereira \& Berbert (2008).

Apesar de inicialmente o relatório receber algumas críticas, foi possível direcionar para os principais gargalos existentes nas unidades. E uma das sugestões foi a criação de um Sistema de Avaliação Institucional para que as unidades fossem avaliadas distintamente, conforme estabelece o MCT/CGEE (2002, pp.147). "Um dos maiores desafios das instituições de Ciência e Tecnologia está no aprimoramento contínuo do seu modelo de gestão e na transparência dos assuntos de interesse público". Marcelino, Matias-Pereira \& Berbert (2008).

Assim, tanto para as Unidades de Pesquisas (UPs) como para as Organizações Sociais (OSs) são estabelecidas metas de desempenho. A preocupação com os serviços prestados, que atendam a sociedade é primordial, pois, apesar de não ser considerado um modelo ideal como alguns autores 
tratam em seus estudos, vem contribuindo muito com a administração pública principalmente por guiar-se pelos princípios da gestão privada. Sano, (2003).

Sano (2003) comenta que o Plano de Reforma do Estado trouxe para o governo diversas vantagens no desenvolvimento das UPs e OSs, mas, na sua visão, uma das principais foi a aplicação da avaliação de desempenho em que se busca atingir metas que aparecem nos termos de compromisso e no contrato de gestão.

\subsection{Teoria da Agência}

De acordo com Klann et al (2009) a Teoria da Agência parte do princípio de que nas relações contratuais existem dois entes chamados de Principal e Agente, o primeiro se configura como um sujeito ativo enquanto o outro passivo. Assim, numa relação contratual o Agente realiza determinada tarefa esperando uma compensação do Principal.

Sendo assim, o principal é caracterizado pelo domínio das informações de cunho estratégico e político, definindo os objetivos para as ações dos agentes, os quais estão ligados ao processo de operacionalização das demandas da equipe, aos processos organizacionais e às especificidades técnicas. Freitas, (2005).

Desta forma, o contrato de gestão nas unidades de pesquisas e organizações sociais foi estabelecido como um sistema de compensação por parte do principal para motivar e monitorar o agente de acordo com seus interesses. Assim, fica evidente que os incentivos são uma forma de monitorar (controlar) os agentes envolvidos a partir de um desempenho observado. Braga; Lima \& Dias, (2007).

Fontes Filho (2003) enfatiza que as aplicações da teoria da agência estão voltadas para as empresas estatais do setor produtivo ou, de forma mais ampliada, para os contratos de gestão. Em âmbito estatal, esse problema fica evidente quando os governantes não conseguem administrar diretamente todos os órgãos do aparelho do estado e delegam essa tarefa a dirigentes que, conforme expressados na teoria da agência, possuem interesses próprios. Em muitos casos, torna-se difícil o processo de avaliação de desempenho em virtude da situação temporal, no entanto, quando se estabelece o contrato de gestão, esse horizonte é minimizado em função dos dirigentes precisarem atingir metas dentro de suas unidades de pesquisas, facilitando, de certo modo, uma avaliação de desempenho da sua gestão.

Por muitos anos, na Administração Pública, predominou uma estrutura que não atendia aos anseios da Sociedade; portanto a necessidade de uma constante renovação em seu ambiente 
organizacional fez com que o uso de medidas de desempenho passasse a estar presente no setor público. Desta forma, pode-se afirmar que o interesse pelo uso dos modelos de avaliação de desempenho está sendo cada vez mais frequente na atividade pública. Marcelino, (1988); MatiasPereira, (2008a/b); Ferlie et al., (1999).

\section{METODOLOGIA DA PESQUISA}

Inicialmente, foi realizada uma pesquisa bibliográfica explorando a Administração Pública e o Modelo de Avaliação de Desempenho Sink \& Tuttle (1993), em seguida tem-se uma breve visão das Unidades compostas por 13 (treze) Unidades de Pesquisas e 04 (quatro) Organizações Sociais ligadas ao Ministério da Ciência e Tecnologia e por fim o uso da teoria da agência para dar sustentação teórica ao estudo. Todo esse conteúdo já foi apresentado nas partes anteriores deste trabalho.

Foram selecionados os gestores das UPOS representados pelos: Diretores, Coordenadores, Analistas, Tecnologistas, Chefe de Divisão e Pesquisadores. No total foram respondidos 142 (cento e quarenta e dois) questionários.

Uma das principais questões é no tocante a seleção das variáveis que foram escolhidas no modelo, como já referendado na fundamentação teórica, o modelo de Sink e Tuttle possuem sete dimensões, destas, foram escolhidas cinco, a saber: Eficácia, Eficiência, produtividade, Inovação e Qualidade. Em seguida, foram determinadas as variáveis, em que se explorou um quantitativo de 25 (vinte e cinco) variáveis, tendo como base os termos de compromisso e os contratos de gestão firmados entre as Unidades de Pesquisas e Organizações Sociais e o Ministério da Ciência e Tecnologia.

Quadro 01 - Construtos dos modelos com as suas variáveis discriminadas

\begin{tabular}{|c|c|}
\hline Construtos & Variáveis \\
\hline \multirow[t]{5}{*}{ 1. Eficácia } & V5. A sua unidade proporciona relações eficazes com outras unidades. \\
\hline & V6. A unidade mantém uma força de trabalho adequada para atingir os objetivos. \\
\hline & V18. $\mathrm{N}^{\mathrm{o}}$ de publicações por colaboradores. \\
\hline & V19. $\mathrm{N}^{\circ}$ de publicações em periódicos indexados no SCI. \\
\hline & V25. Velocidade no desenvolvimento de novos produtos. \\
\hline \multirow{5}{*}{ 2.Eficiência } & V4. Existe um equilíbrio entre as medidas de eficiência e efetividade do processo na unidade. \\
\hline & V9. Eficiência no trabalho. \\
\hline & V16. Processos e Técnicas Desenvolvidas. \\
\hline & V21. Qualidade e eficiência nos processos. \\
\hline & V24. Capacidade de Inovação. \\
\hline \multirow[t]{5}{*}{ 3.Produtividade } & $\begin{array}{l}\text { V2. A Unidade de Pesquisa está com nível máximo de sua capacidade no tocante a sua } \\
\text { produção cientifica e/ou produção tecnológica. }\end{array}$ \\
\hline & V7. A unidade faz uso produtivo e eficiente dos recursos disponíveis. \\
\hline & V13. Capacitação e Treinamento. \\
\hline & V14. Produtividade. \\
\hline & V15. Índice Geral de Publicação \\
\hline
\end{tabular}

Revista de Administração e Inovação, São Paulo, v. 11, n.2, p. 51-70, abr./jun. 2014. 


\begin{tabular}{|l|l|}
\hline 4.Inovação & $\begin{array}{l}\text { V1. A Unidade de Pesquisa possui um grau de contribuição como ferramenta de } \\
\text { autodesenvolvimento e transferência de conhecimento. }\end{array}$ \\
\hline & V10. Introdução de novos produtos. \\
\hline & V11. Tecnologia de produto. \\
\hline & V12. Gerenciamento da inovação. \\
\hline & V17. Aplicação em P\&D(Pesquisa e Desenvolvimento). \\
\hline & V23. Transferência de tecnologia para a sociedade. \\
\hline & V3. Existe o uso de medidas alinhadas a qualidade do processo. \\
\hline & V8. Qualidade. \\
\hline & V20. Produção de relatórios finais. \\
\hline & V22. Aspectos relacionados a inclusão social. \\
\hline
\end{tabular}

Fonte: Os autores, 2011.

Utilizou-se como instrumento de pesquisa um questionário, que, segundo Silva e Menezes (2001, pp. 33) "é uma série ordenada de perguntas que devem ser respondidas por escrito pelo informante". Os questionários utilizados foram do tipo estruturados, compostos por questões fechadas. Gil (1999, pp. 92) afirma que "a entrevista estruturada é quando se desenvolve a partir de uma relação fixa de perguntas". Por fim, de acordo com Chizzotti (1991, pp. 57) “é um tipo de comunicação entre um pesquisador que pretende colher informações sobre fenômenos e indivíduos que detenham essas informações e possam emiti-las".

O questionário foi dividido em três blocos, subdivididos em partes menores, os quais expressam e contempla o modelo de avaliação de desempenho de Sink e Tuttle.

No Bloco I foram tratadas as principais informações gerais sobre os respondentes: Gênero, Faixa Etária, Grau de Instrução, Unidade de Pesquisa e/ou Organizações Sociais e Posição Funcional.

Para o Bloco II são apresentadas 07 assertivas. Em que se busca expressar as opiniões dos respondentes com base numa escala de 1 a 5 , em que 1 é discordo totalmente, 2 discordo parcialmente, 3 neutro, 4 concordo parcialmente e 5 concordo totalmente.

No Bloco III foram tratadas 18 variáveis que utilizam mais especificamente os indicadores inerentes às três categorias propostas no Termo de Compromisso, celebradas entre as Unidades de Pesquisas e o MCT: Nacionais (aplicados a todas as Unidades); Focais (específicos para grupos de unidades com atuação em áreas similares de Ciência \& Tecnologia); e Institucionais (exclusivos de cada Unidade e por elas propostos). Desta forma, neste bloco foi evidenciada a percepção individual dos respondentes aos fatores de desempenho direcionados a cada ação desenvolvida pela unidade de pesquisa em questão. Assim, os principais fatores são apresentados numa coluna central, em cada lado da coluna central existe uma escala com números de 1 a 5 para que o respondente expresse sua opinião a respeito delas. Entre as principais áreas são levantadas duas colunas a da esquerda é chamada de "Grau de Importância de Melhoria para a Unidade de Pesquisa", e na direita "Efeito destas Medidas sobre Melhorias na Unidade de Pesquisa". 
Os dados deste estudo coletados através dos questionários foram compilados com o auxílio do Microsoft Office Excel®, no qual foram confeccionados as tabelas da faixa etária, gênero, entre outros. O uso do software Statiscical Package for the Social Sciences (SPSS) possibilita o tratamento dos testes estatísticos.

Segundo Martins \& Theóphilo (2007), a Escala Likert foi desenvolvida nos anos 30 por Rensis Likert. É muito usada nas pesquisas na área de Ciências Sociais e trata de um conjunto de itens em forma de afirmações numa escala de um a cinco, ou sete. Neste estudo, a escala utilizada é a Likert e foi tratado numa escala de 1 a 5 com 07 afirmações na primeira parte e 18 na parte dois.

Desta forma, a análise estatística segue a seguinte estrutura:

Quanto às variáveis foram nominais, apenas na parte tratada como identificação (Gênero, faixa etária, grau de instrução, unidade de pesquisa do respondente e posição funcional), e, em um segundo momento, todas as variáveis serão do tipo ordinárias. Vieira (2007, pp. 94) afirma que "se uma variável é ordinal, espera-se que as diferenças entre os grupos sejam explicadas pela ordenação".

No tocante ao tratamento estatístico, inicialmente foi feita a Estatística Descritiva abordando análise de frequência, as Medidas de Localização ou de Tendência Central; Medidas Separatrizes ou Ordenamento; e Medidas de Dispersão ou Variabilidade. Em seguida foi feito o Qui-Quadrado de Pearson para Independência. Por fim, para identificar as correlações existentes entre as variáveis, foi utilizada a Correlação de Spearman.

O universo da pesquisa, de acordo com Silva (2003), é um conjunto que representa os indivíduos do estudo, enquanto a amostra é seu subconjunto. Sendo assim, o universo da pesquisa é formado pelas 17 unidades do Ministério da Ciência e Tecnologia. Assim, fez-se uso da estratificação, que, de acordo com Vergara (2000, pp. 45) é "uma parte do universo escolhido, segundo algum critério de representatividade". Pois, existiam outras unidades, mesmo assim concentrou-se nas unidades que realizaram o seu alinhamento estratégico.

Para Matias-Pereira (2007), Beuren (2006) e Silva (2003), os métodos de amostragem podem ser de dois tipos: probabilísticos e não probabilísticos. Diante disto, o estudo terá foco em dados não probabilísticos que leva em consideração o julgamento do pesquisador, a acessibilidade dos dados e a conveniência da pesquisa. Assim, partindo do alinhamento estratégico consolidado das unidades no MCT e do acesso junto ao coordenador-geral das unidades de pesquisas, a pesquisa se configura do tipo não probabilística, por conveniência e por acessibilidade. 


\section{ANALISE DOS RESULTADOS}

Os resultados apresentados foram com base nos questionários divididos em duas partes na primeira têm-se sete questões e na segunda parte trabalhou-se sob dois enfoques: o grau de importância e o efeito das variáveis nas UPOS as outras 18 questões.

Em seguida, foi feita uma análise descritiva dos dados. Vale salientar que nesta análise foram utilizados apenas os valores extremos, por isso que, quando somadas às frequências das questões, não se chegará a uma totalidade de $100 \%$. Ou seja, os percentuais intermediários foram eliminados.

Analisando a tabela 01 de frequência abaixo, as variáveis que apresentaram maiores níveis de concordância foram:

1. As unidades de pesquisas possuem um grau de contribuição como ferramenta de autodesenvolvimento e transferência de conhecimento; e

2. A unidade proporciona relações eficazes com outras unidades;

Pode-se dizer que nesses primeiros resultados, as unidades de pesquisas, estão preocupadas com ferramentas de autodesenvolvimento e transferência de conhecimento que em suma contribui para o progresso cientifico, uso produtivo e eficiente dos recursos que estão sendo disponibilizados para as UPOS.

Tabela 01 - Análise de Frequência do Grau de Concordância

\begin{tabular}{|c|c|c|}
\hline ASSERTIVAS & $\begin{array}{l}\text { DISCORDÂACIA } \\
(\%)\end{array}$ & $\begin{array}{c}\text { CONCORDÂNCIA } \\
(\%)\end{array}$ \\
\hline $\begin{array}{lccc}\text { A Unidade de } & \text { Pesquisa } & \text { possui um grau } & \text { de } \\
\text { contribuição } & \text { como } & \text { ferramenta } & \text { de } \\
\text { autodesenvolvimento } & \text { e } & \text { transferência } & \text { de } \\
\text { conhecimento. } & & & \end{array}$ & 8,5 & 75,3 \\
\hline $\begin{array}{l}\text { A Unidade de Pesquisa está com nível máximo de sua } \\
\text { capacidade no tocante a sua produção cientifica e/ou } \\
\text { produção tecnológica. }\end{array}$ & 33,1 & 47,2 \\
\hline $\begin{array}{l}\text { Existe o uso de medidas alinhadas a qualidade do } \\
\text { processo }\end{array}$ & 33,1 & 38,8 \\
\hline $\begin{array}{l}\text { Existe um equilíbrio entre as medidas de eficiência e } \\
\text { efetividade do processo na unidade }\end{array}$ & 31 & 37,3 \\
\hline $\begin{array}{l}\text { A sua unidade proporciona relações eficazes com } \\
\text { outras unidades }\end{array}$ & 11,3 & 71,9 \\
\hline $\begin{array}{l}\text { A unidade mantém uma força de trabalho adequada } \\
\text { para atingir os objetivos }\end{array}$ & 68,3 & 22,5 \\
\hline $\begin{array}{l}\text { A unidade faz uso produtivo e eficiente dos recursos } \\
\text { disponíveis }\end{array}$ & 13,4 & 69,1 \\
\hline
\end{tabular}

Fonte: Dados da Pesquisa, 2011. 
No tocante ao grau de discordância, a principal variável foi: A unidade mantém uma força de trabalho adequada para atingir os objetivos. De acordo com o que foi observado, as unidades de pesquisas não mantêm uma força de trabalho adequada; há a percepção de que todas as unidades estão sobrecarregadas necessitando de um maior apoio de pessoal; isso fica mais evidente no momento em que se fala na qualidade do processo desenvolvido por cada unidade, principalmente no tocante à produção científica, quando os respondentes afirmam que as unidades não atingiram sua capacidade máxima e que, no curto espaço de tempo, não se tem uma previsão de melhoria, já que não existe uma visão crítica para aperfeiçoar este processo.

Após a análise do grau de concordância, foram analisadas as 18 variáveis da segunda parte que tratam de dois enfoques. O primeiro a respeito da perspectiva de grau de importância das variáveis para as unidades e o segundo a respeito do efeito que estas variáveis causam em cada unidade.

Tabela 02 - Grau de Importância das Variáveis nas Unidades de Pesquisas

\begin{tabular}{|c|c|c|}
\hline GRAU DE IMPORTÂNCIA & $\begin{array}{c}\text { SEM } \\
\text { IMPORTÂNCIA } \\
(\%)\end{array}$ & $\begin{array}{c}\text { MUITO } \\
\text { IMPORTANTE } \\
(\%)\end{array}$ \\
\hline Qualidade & 3,5 & 94,4 \\
\hline Eficiência no trabalho & 4,9 & 95,1 \\
\hline Introdução de novos produtos & 16,9 & 59,2 \\
\hline Tecnologia de produto & 18,3 & 61,9 \\
\hline Gerenciamento da inovação & 14,8 & 71,2 \\
\hline Capacitação e Treinamento & 4,9 & 86,6 \\
\hline Produtividade & 3,5 & 85,3 \\
\hline Índice Geral de Publicação & 4,2 & 87,4 \\
\hline Processos e Técnicas Desenvolvidas & 7 & 71,9 \\
\hline Aplicação em P\&D(Pesquisa e Desenvolvimento) & 2,8 & 82,9 \\
\hline $\mathrm{N}^{\circ}$ de publicações por colaboradores & 12,7 & 68,3 \\
\hline $\mathrm{N}^{\mathrm{o}}$ de publicações em periódicos indexados no SCI & 9,2 & 76,8 \\
\hline Produção de relatórios finais & 12,7 & 64,8 \\
\hline Qualidade e eficiência nos processos & 4,2 & 85,9 \\
\hline Aspectos relacionados a inclusão social & 16,9 & 68,3 \\
\hline Transferência de tecnologia para a sociedade & 13,4 & 72,6 \\
\hline Capacidade de Inovação & 8,5 & 74 \\
\hline Velocidade no desenvolvimento de novos produtos & 23,2 & 56,3 \\
\hline
\end{tabular}

Fonte: Dados da Pesquisa, 2011.

As duas variáveis mais importantes foram: Eficiência no trabalho e Qualidade. Para as unidades de pesquisas, a eficiência no trabalho é um dos fatores mais importantes, seguido da qualidade e da necessidade que elas precisam para desenvolver seus trabalhos por meio de uma boa infraestrutura.

Por outro lado, na parte negativa, ou seja, as variáveis menos importantes para os respondentes foram as seguintes: 
1. Velocidade no desenvolvimento de novos produtos; e

2. Tecnologia de produto;

Acredita-se que estas variáveis representam os principais gargalos das unidades de pesquisas. Apesar de serem organizações que estão voltadas para a área de tecnologia, elas apresentam deficiência no desenvolvimento de novos produtos, ocasionada pela falta de tecnologia para avançarem em algumas pesquisas, e principalmente, sofrem fortes influências da burocracia pública.

Observando a tabela 03, abaixo, em que se analisa o efeito apresentado pelas variáveis nas unidades de pesquisas, foram encontradas as seguintes respostas:

1. Aplicação em P\&D (Pesquisa e Desenvolvimento); e

2. Índice Geral de Publicação

Tabela 03 - Qual o Efeito das Variáveis nas Unidades de Pesquisas

\begin{tabular}{|c|c|c|}
\hline QUAL O EFEITO & $\begin{array}{c}\text { SEM EFEITO } \\
(\boldsymbol{\%})\end{array}$ & $\begin{array}{c}\text { COM MUITO EFEITO } \\
(\mathbf{\%})\end{array}$ \\
\hline Qualidade & 11,3 & 67,6 \\
\hline Eficiência no trabalho & 12 & 67,6 \\
\hline Introdução de novos produtos & 23,9 & 42,3 \\
\hline Tecnologia de produto & 19 & 45,8 \\
\hline Gerenciamento da inovação & 27,5 & 48,6 \\
\hline Capacitação e Treinamento & 12,7 & 66,2 \\
\hline Produtividade & 11,3 & 67,6 \\
\hline Índice Geral de Publicação & 6,3 & 69,7 \\
\hline Processos e Técnicas Desenvolvidas & 16,2 & 59,8 \\
\hline Aplicação em P\&D(Pesquisa e Desenvolvimento) & 9,2 & 72,5 \\
\hline $\mathrm{N}^{\mathbf{o}}$ de publicações por colaboradores & 16,2 & 52,1 \\
\hline $\mathrm{N}^{\text {o }}$ de publicações em periódicos indexados no SCI & 18,3 & 59,9 \\
\hline Produção de relatórios finais & 16,2 & 57,1 \\
\hline Qualidade e eficiência nos processos & 13,4 & 58,5 \\
\hline Aspectos relacionados a inclusão social & 19,7 & 50,7 \\
\hline Transferência de tecnologia para a sociedade & 21,8 & 58,5 \\
\hline Capacidade de Inovação & 16,2 & 54,3 \\
\hline Velocidade no desenvolvimento de novos produtos & 31,7 & 40,9 \\
\hline
\end{tabular}

Fonte: Dados da Pesquisa, 2011.

Como se pode observar, existiram duas variáveis que se destacam e mostram o quão é importante para os respondentes a questão de publicação nas unidades, isso se torna mais evidente nas unidades de pesquisa voltadas para área de tecnologia.

Logo abaixo, foi calculada a estatística descritiva. Inicialmente foi analisado o Modelo de Sink e Tuttle com base nos quatro construtos especificados no modelo. Assim, para a dimensão Eficácia, na variável 19G, que trata do grau de importância que foi dado pelos respondentes com relação ao $n^{\circ}$ de 
publicações em periódicos SCI, obteve-se média 4,13 com a moda e mediana respectivamente 5,00 e 4,5 e, para os três quartis, as respostas foram tratadas como uma das mais importantes pelos respondentes. E com uma média menor 2,30, a variável V6 frisa bem a falta de recursos humanos nas unidades, ou seja, para os respondentes, as unidades não mantêm uma força de trabalho adequada para atingir seus objetivos.

Tabela 04 - Estatística Descritiva do Modelo Sink e Tutlle

\begin{tabular}{|c|c|c|c|c|c|c|c|c|c|c|c|c|}
\hline \multirow{2}{*}{$\begin{array}{c}\text { Construtos } \\
\text { CONST } \\
\end{array}$} & \multirow{2}{*}{$\begin{array}{c}\text { Variáveis } \\
\text { VAR } \\
\end{array}$} & \multicolumn{3}{|c|}{$\begin{array}{l}\text { Medidas de Tendência } \\
\text { Central }\end{array}$} & \multicolumn{3}{|c|}{$\begin{array}{l}\text { Medidas } \\
\text { Separatrizes }\end{array}$} & \multicolumn{3}{|c|}{$\begin{array}{l}\text { Medidas de } \\
\text { Dispersão }\end{array}$} & \multicolumn{2}{|c|}{$\begin{array}{l}\text { Medidas de } \\
\text { Assimetria e } \\
\text { Curtose }\end{array}$} \\
\hline & & MED & MDA & MNA & $\mathrm{Q}_{1}$ & $\mathrm{Q}_{2}$ & $\mathrm{Q}_{3}$ & AT & DP & $S^{2}$ & ASS & CUR \\
\hline \multirow[t]{2}{*}{ EFICÁCIA } & V6 & 2,30 & 2 & 2 & 1 & 2 & 3 & 4 & 1,254 & 1,586 & 0,764 & $-0,561$ \\
\hline & V19G & 4,13 & 5 & 4,5 & 4 & 4,5 & 5 & 4 & 1,100 & 1,209 & $-1,276$ & 0,970 \\
\hline \multirow[t]{2}{*}{ EFICIÊNCIA } & V4 & 2,99 & 3 & 3 & 2 & 3 & 4 & 4 & 1,123 & 1,262 & $-0,307$ & $-0,750$ \\
\hline & V9G & 4,62 & 5 & 5 & 4 & 5 & 5 & 3 & 0,671 & 0,450 & $-2,091$ & 4,841 \\
\hline \multirow{2}{*}{$\begin{array}{l}\text { PRODUTIVI } \\
\text { DADE }\end{array}$} & V2 & 3,11 & 4 & 3 & 2 & 3 & 4 & 4 & 1,233 & 1,519 & $-0,310$ & -1 \\
\hline & V15G & 4,41 & 5 & 5 & 4 & 5 & 5 & 4 & 0,843 & 0,711 & $-1,542$ & 2,224 \\
\hline \multirow[t]{2}{*}{ INOVAÇÃO } & V10E & 3,23 & 3 & 3 & 3 & 3 & 4 & 4 & 1,183 & 1,400 & $-0,280$ & $-0,630$ \\
\hline & V17G & 4,39 & 5 & 5 & 4 & 5 & 5 & 4 & 0,875 & 0,765 & $-1,508$ & 2,182 \\
\hline \multirow[t]{2}{*}{ QUALIDADE } & V3 & 3,20 & 4 & 3 & 2 & 3 & 4 & 4 & 1,246 & 1,554 & $-0,217$ & -1 \\
\hline & V8G & 4,71 & 5 & 5 & 5 & 5 & 5 & 4 & 0,740 & 0,547 & $-3,212$ & 11,086 \\
\hline
\end{tabular}

Fonte: Dados da Pesquisa, 2011.

LEGENDA

CONST $=$ Construto VAR $=$ Variável $\quad$ MED $=$ Média $\quad \mathrm{MDA}=$ Moda

$\mathrm{MNA}=$ Mediana $\mathrm{Q}_{1}=1^{\circ}$ Quartil $\mathrm{Q}_{2}=2^{\circ}$ Quartil $\mathrm{Q}_{3}=3^{\circ}$ Quartil AT $=$ Amplitude total

$\mathrm{DP}=$ Desvio padrão $\mathrm{S}^{2}=$ Variância ASS $=$ Coeficiente de Assimetria

CUR $=$ Coeficiente de Curtose

No construto de Eficiência, teve-se como maior média a exigência da eficiência no trabalho com média 4,62, que por sinal foi a maior média do modelo. Já no lado oposto, a variável que teve a menor média foi o equilíbrio entre as medidas de eficiência e a efetividade destas ações; pelo que se pode observar, existe uma eficiência nas unidades, no entanto falta a efetividade do processo. Isso pode estar ocorrendo em função da falta de percepção dos respondentes que não enxergam que o planejamento estratégico foi elaborado dentro de uma perspectiva em que se tinha uma proposta a ser seguida com o fechamento do ciclo. Agora será mais fácil realizar uma reestruturação deste plano com as alterações de melhorias.

Para o construto Produtividade, a variável que apresentou maior média foi: Índice Geral de Publicação, com 4,41, o que mostra uma preocupação por parte das unidades em apresentar suas pesquisas para a Sociedade, corroborando assim para um melhor desenvolvimento de tecnologias. Contudo, mesmo com alto grau de importância dada a questões como publicações, as unidades de pesquisas afirmam que elas não estão com sua capacidade máxima de produção científica e/ou tecnológica, apresentando para essa variável uma média menor, de 3,11.

Revista de Administração e Inovação, São Paulo, v. 11, n.2, p. 51-70, abr./jun. 2014. 
No tocante ao construto Inovação, as unidades acreditam que a Introdução de Novos Produtos não tem causado impactos tecnológicos em seus trabalhos, em função disso, a média desta variável foi de 3,23. Entretanto, neste mesmo construto, é dada uma atenção maior para aplicação em P\&D, ou seja, existe um alto grau de importância para esta variável comprovada pela sua média de 4,39.

No construto Qualidade, observam-se as seguintes médias: A variável qualidade, com média 4,71, e o uso de medidas alinhadas à qualidade do processo, com menor média, 3,20.

No que diz respeito às medidas de assimetria e curtose para este modelo, pode-se dizer que a curva é assimétrica negativa e para a maioria da distribuição é do tipo platicúrtica achatada.

Utilizou-se o teste $\chi^{2}$ para verificar a existência de relações entre algumas variáveis e as características estruturais e pessoais nas unidades de pesquisas. $\mathrm{O}$ intuito foi identificar se existe influência no desempenho da unidade no que diz respeito aos níveis de eficácia, eficiência, produtividade, qualidade e accountability.

Para o modelo de Sink e Tutlle, foram testadas as hipóteses abaixo:

H0: Não existe relação entre as variáveis do modelo de Sink e Tuttle com as características estruturais e pessoais da unidade de pesquisa.

H1: Existe relação entre as variáveis do modelo de Sink e Tuttle com as características estruturais e pessoais da unidade de pesquisa.

Tabela 05 - Modelo de Silk e Tutlle

\begin{tabular}{|c|c|c|}
\hline CRUZAMENTOS & Teste $\boldsymbol{\chi}^{\mathbf{2}}$ & P-Valor \\
\hline GI X PROCIT2 & 21,165 & 0,048 \\
\hline GI X EMEFFA4 & 23,796 & 0,022 \\
\hline GI X RELEFI5 & 21,971 & 0,038 \\
\hline GI X FORTRA6 & 22,422 & 0,033 \\
\hline GI X PROREC7 & 22,304 & 0,034 \\
\hline UPOS X INTPRO10E & 85,018 & 0,041 \\
\hline UPOS X CAPTRE13G & 89,226 & 0,020 \\
\hline UPOS X PUBSCI19G & 93,256 & 0,010 \\
\hline UPOS X PUBSCI19E & 90,591 & 0,016 \\
\hline UPOS X FOCPRO24G & 85,089 & 0,040 \\
\hline
\end{tabular}

$\chi_{\text {Crítico }}^{\mathbf{2}}=\mathbf{2 1 , 0 2 6}$ e grau de liberdade=12; GRAU DE INSTRUÇÃO

$\chi_{\text {Crítico }}^{2}=\mathbf{8 3 , 6 7 5}$ e grau de liberdade $=64 ;$ UNIDADE DE PESQUISA

Fonte: Dados da Pesquisa, 2011.

RESULTADO 1: O valor $\chi^{2}=21,165 ; \chi^{2}=23,796 ; \chi^{2}=21,971 ; \chi^{2}=22,422 ; \chi^{2}=22,304$; com 12 graus de liberdade, é significativo $(\mathrm{P}$-valor $=0,048 ; \mathrm{P}$-valor $=0,022 ; \mathrm{P}$-valor $=0,038 ; \quad \mathrm{P}$-valor $=$ 0,033; P-valor $=0,034 ;<5 \%)$. Portanto, ao nível de $5 \%$, pode-se concluir que: A unidade de pesquisa 
não está com nível máximo de sua capacidade; não existe um equilíbrio entre as medidas de eficiência e efetividade no processo; a unidade proporciona relações eficazes com outras unidades; a força de trabalho é insuficiente; e a unidade faz uso produtivo e eficiente dos recursos disponíveis, ou seja, quanto maior o grau de instrução mais influência foi exercida nessas variáveis.

RESULTADO 2: O valor $\chi^{2}$ das outras variáveis; com 12 graus de liberdade, é não significativo (Pvalor > 5\%). Portanto, ao nível de 5\%, não rejeita H0, ou seja, não existe influência entre as outras variáveis e o Grau de Instrução.

RESULTADO 3: O valor $\chi^{2}=85,018 ; \chi^{2}=89,226 ; \chi^{2}=93,256 ; \chi^{2}=90,591 ; \chi^{2}=85,089$; com 64 graus de liberdade, é significativo ( $\mathrm{P}$-valor =0,041; $\mathrm{P}$-valor =0,020; $\mathrm{P}$-valor =0,010; $\mathrm{P}$-valor =0,016; $\mathrm{P}$-valor $=0,040 ;<5 \%)$. Portanto, ao nível de 5\%, pode-se concluir que: A introdução de novos produtos; a capacitação e treinamento; a capacidade de inovação; e o número de publicações em periódicos indexados no SCI influenciam as Unidades de Pesquisas e/ou Organizações Sociais.

RESULTADO 4: O valor $\chi^{2}$ das outras variáveis; com 64 graus de liberdade, é não significativo (Pvalor > 5\%). Portanto, ao nível de 5\%, não rejeita H0, ou seja, não existe influência entre as outras variáveis e a UPOS.

Inicialmente, foi feita uma Correlação de Spearman, na qual foram relacionadas todas as variáveis estudadas no artigo. Todavia, por questões de importância, preferiu-se apresentar apenas as correlações que se fizeram presentes acima de 0,499, pois, são as que possuem maior significância para o trabalho. Destas correlações ainda foram separadas as variáveis que mais se correlacionaram com outras variáveis para um melhor poder de explicação.

Argumenta-se que, os demais coeficientes foram abaixo de 0,500 ou ainda não significativos para os níveis de significância de 0,05 e 0,01, por isso não foram apresentados. Assim, toma-se como premissa que não se pode rejeitar a hipótese nula, ou seja, não existe associação entre os demais cruzamentos das variáveis.

Na tabela 06, foi apresentada a Correlação de Spearman com relação à variável QUAFIO21E, que faz parte do modelo Sink e Tuttle e está inserida no construto Eficiência. Pode-se afirmar, portanto, que, das variáveis estudadas, essas foram as que mais se relacionaram, ou seja, Qualidade e Eficiência nos Processos estão associadas positivamente com 5 variáveis, são elas:

Revista de Administração e Inovação, São Paulo, v. 11, n.2, p. 51-70, abr./jun. 2014. 
1. Eficiência no Trabalho;

2. Capacitação e Treinamento;

3. Processos e Técnicas desenvolvidas;

4. Capacidade de Inovação; e

5. Velocidade no desenvolvimento de novos produtos;

Tabela 06 - Correlação de Spearman da variável QUAFIO21E

\begin{tabular}{|c|c|c|c|c|c|c|}
\hline \multicolumn{2}{|c|}{ Variáveis } & V9 & V13 & V16 & V24 & V25 \\
\hline QUAFIO21E & Coeficiente & 0,562 & 0,500 & 0,500 & 0,554 & 0,600 \\
\hline & Sig. & 0,000 & 0,000 & 0,000 & 0,000 & 0,000 \\
\hline
\end{tabular}

*A correlação é significativa no nível de significância de 0,01 .

Fonte: Dados da Pesquisa, 2011.

Essas correlações mostram que a qualidade das unidades está associada à eficiência no trabalho, o que revela uma preocupação em capacitar e treinar seu pessoal por meio de processos e técnicas desenvolvidas, com isso espera-se que as unidades alcancem capacidade de inovação, velocidade no desenvolvimento de novos produtos e estabeleça ações estratégicas para atender os principais pontos do plano diretor estabelecidos entre o MCT e as UPOS.

Para tabela 07, a variável EFITRA9E faz parte do Modelo de Sink e Tuttle e do construto Eficiência e essa variável está relacionada positivamente com 4 variáveis que são elas:

1. Qualidade;

2. Capacitação e Treinamento;

3. Produtividade; e

4. Qualidade e eficiência nos processos;

Tabela 07 - Correlação de Spearman da variável EFITRA9E

\begin{tabular}{|l|l|l|l|l|l|}
\hline Variáveis & & V8 & V13 & V14 & V21 \\
\hline EFITRA9E & Coeficiente & 0,631 & 0,515 & 0,604 & 0,562 \\
\hline & Sig. & 0,000 & 0,000 & 0,000 & 0,000 \\
\hline
\end{tabular}

*A correlação é significativa no nível de significância de 0,01 .

Fonte: Dados da Pesquisa, 2011.

De acordo com o que é apresentado na tabela 07, as variáveis qualidade e produtividade estão associadas positivamente com eficiência no trabalho, ou seja, o modelo de Sink e Tuttle, no tocante ao construto eficiência, mostra que as unidades de pesquisas se preocupam com a avaliação de 
desempenho porque tem nas variáveis mais bem correlacionadas, qualidade, produtividade e accountability.

Sintetizando as últimas tabelas apresentadas com base na Correlação de Spearman, pode-se inferir que as UPOS estão preocupadas em apresentar um nível de qualidade, com base na eficiência dos resultados, ou seja, a produtividade é um fator preponderante e sem dúvidas o processo de alinhamento estratégico é levado a sério por elas.

Quando se fala em avaliação de desempenho é correto afirmar que as unidades de pesquisas estão preocupadas em atingir as metas estabelecidas no plano diretor do MCT e que as principais medidas estão alinhadas à qualidade do processo, possuindo uma visão crítica para aperfeiçoar o processo e a busca de um equilíbrio entre eficiência e efetividade.

\section{CONSIDERAÇÕES FINAIS}

É perceptivo que algumas unidades de pesquisas estão mais preocupadas em atingir suas metas de pesquisas, com o intuito de conseguir mais bolsas e até mesmo mais recursos, do que com o alinhamento estratégico.

A análise também deixa claro que o teste $\chi^{2}$ elencou e estruturou as principais variáveis com relação às características pessoais e estruturais das unidades, nas quais se tem uma preocupação com a produtividade no tocante a publicações e níveis tecnológicos, bem como, uma visão crítica para o aperfeiçoamento do processo e uso de medidas alinhadas à qualidade.

No tocante às variáveis mais importantes, as duas principais foram eficiência no trabalho e qualidade, reforçando a idéia de que as características das unidades influenciam o desempenho das unidades.

O teste $\chi^{2}$ mostra para o modelo de Sink e Tuttle que o gênero influencia os níveis de produtividade nas unidades de pesquisas. O maior número de publicações destacou-se na área de Ciências Exatas e da Natureza e na área das Engenharias, principalmente com as unidades (INPE, CBPF e CETEM), ou seja, quanto maior o nível de tecnologia das unidades maior o número de publicação.

É consenso entre as unidades que a força de trabalho está aquém das necessidades de cada unidade; todas elas enfatizaram que os números de servidores precisam ser aumentados e que ainda não estão em sua capacidade máxima de produção.

Dos construtos utilizados do modelo os que mais se destacaram foram Eficiência, Inovação e Qualidade.

Revista de Administração e Inovação, São Paulo, v. 11, n.2, p. 51-70, abr./jun. 2014. 
Pode-se afirmar que os procedimentos de avaliação de desempenho com base no plano diretor e nos termos de compromissos e/ou contratos de gestão considerados pelas unidades do MCT foram estabelecidos e o objetivo alcançado.

\section{REFERÊNCIAS BIBLIOGRÁFICAS}

Ghalayini, A. M.; Noble, J. (1996). The changing basis of performance measurement. International Journal of Operations \& Production Management, Vol. 16 No. 8, pp. 63-80.

Rolstadas, A. (1998). Enterprise performance measurement. International Journal of Operations \& Production Management, Vol. 18 No. 9/10, pp. 989-999.

Taticchi, P.; Balachandran, K. R. (2008). Research Forward performance measurement and management integrated frameworks. International Journal of Accounting and Information Management, vol. 16 No. 2, pp. 140-154.

Foster, G.; Young, S. M. (1997). Frontiers of management accounting research. Journal of Management Accounting Research, 9, pp. 63-77.

Ittner, C. D.; Larcker, D. F. (1998). Innovations in performance measurement: trends and research implications. Journal of Management Accounting Research, pp. 205-238.

Da Paz, P. H. F. (2003). Um Estudo da Avaliação da Gestão Pública Baseada na Experiência de Governos Estrangeiros com a Utilização do Balanced Scorecard. Programa de Pós-Graduação em Engenharia de Produção. Dissertação de Mestrado da Universidade Federal de Pernambuco - UFPE.

Sink, D. Scott; Tuttle, Thomas C. (1993). Planejamento e medição para a performance. Tradução: Elenice Mazzilli e Lúcia F. Silva. Rio de Janeiro: Qualitymark. Tradução de: Planning and measurement in your organization of the future.

Schmidt, P.; Santos, J. L. dos; Martins, M. A. (2006). Avaliação de Empresas: Foco na Análise de Desempenho para o Usuário Interno. São Paulo: Atlas.

Marcelino, G. F; Matias-Pereira, J; Bebert, C. O. (2008). Gestão Estratégica em Ciência e Tecnologia: Avaliação de uma Experiência de Alinhamento Estratégico no Governo Federal. In: Mendonça, Gilson Martins (org). Estudos Contemporâneos em Organização e Gestão. São Luiz. Editora UEMA.

MCT. (2009). Ministério da Ciência e Tecnologia. Recuperado em Agosto 2009. <http:www.mct.gov.br>

MCT/CGEE. 2002. Memória da Conferência Nacional de Ciência, Tecnologia e Inovação.

Sano, H. (2003). Nova Gestão Pública e Accountability: O caso das Organizações Sociais Paulistas. Dissertação de Mestrado do Programa de Pós-Graduação da Fundação Getúlio Vargas da Escola de Administração de Empresas de São Paulo - FGV/EAESP.

Klann, R. C; Gomes, E. do C. O; Greuel, M. A.; Bezerra, F. A. (2009). Influência do Risco Moral e da Accountability nas Tomadas de Decisões. ANPCONT. 
Freitas, L. C. (2005). TV Digital no Brasil: A Evolução do Processo Decisório à Luz de uma Perspectiva Organizacional de Teorias Econômicas. In: ENCONTRO NACIONAL DOS PROGRAMAS DE PÓS-GRADUAÇÃO E ADMINISTRAÇÃO, 29, 2005, Brasília. Anais. Brasília: ANPAD.

Braga, C.D.; Lima, R.E.; Dias, C.G; (2007). A teoria da agência e os recursos humanos nas organizações. In: ENCONTRO NACIONAL DOS PROGRAMAS DE PÓS-GRADUAÇÃO E ADMINISTRAÇÃO, 31, 2007, Rio de Janeiro. Anais. Rio de Janeiro: ANPAD.

Fontes Filho, J. R. (2003). Governança Organizacional Aplicada ao Setor Público. VIII Congresso Internacional Del CLAD sobre La Reforma Del Estado Y de La Administración Pública, Panamá, 28 31 Oct.

Marcelino, G. F. (1988). Governo, imagem e sociedade. Brasília: Fundação Centro de Formação do Servidor Público - FUNCEP.

Matias-Pereira, J. (2008a). Curso de Administração Pública Foco nas Instituições e Ações Governamentais. São Paulo: Atlas.

Matias-Pereira, J. (2008b). Manual de Gestão Pública Contemporânea. São Paulo: Atlas.

Ferlie, E.; Asburner, L.; Fitzgerald, L.; Pettigrew, A. (1999). A Nova Administração Pública em Ação. Tradução de Sara Rejane de Freitas Oliveira; revisão técnica de Tomás de Aquino Guimarães. Brasília, Editora Universidade de Brasília: ENAP.

Silva, Edna Lúcia da; Menezes, Estera Muskat. (2001). Metodologia da pesquisa e elaboração de dissertação. 3. ed. rev. e atual. Florianópolis: Laboratório de Ensino à Distância da UFSC.

Gil, A. C. (1999). Métodos e Técnicas de Pesquisa Social. São Paulo. Atlas.

Chizzotti, A. (1991). A Pesquisa em Ciências Humanas e Sociais. São Paulo: Cortez.

Martins, G de A.; Theóphilo, C. R. (2007). Metodologia da investigação científica para Ciências Sociais Aplicadas. São Paulo: Atlas.

Vieira, S. (2003). Bioestatística: tópicos avançados. Rio de Janeiro: Campus.

Silva, A. C. R. da. (2003). Metodologia da Pesquisa Aplicada à Contabilidade. São Paulo: Atlas.

Vergara S. (2000). Projetos e relatórios de pesquisa em administração. São Paulo: Ed. Atlas.

Matias-Pereira, J. (2007). Manual de Metodologia da Pesquisa Científica. São Paulo: Atlas.

Beuren, I. M. (2006). Como elaborar trabalhos monográficos em contabilidade: Teoria e prática. 3 ed. São Paulo: Atlas. 


\title{
PERFORMANCE EVALUATION IN THE MINISTRY OF SCIENCE AND TECHNOLOGY: A STUDY OF MODEL SINK AND TUTTLE
}

\begin{abstract}
The main objective of this paper is to verify that the Model Performance Evaluation of Sink and Tuttle can be employed in the Ministry of Science and Technology (MCT). The research methodology is descriptive and exploratory. The Sink and Tuttle model constructs have seven of these, five were chosen, namely: Effectiveness, Efficiency, Productivity, Innovation and Quality. In total 142 were returned (one hundred forty-two) questionnaires. The test $\chi^{2}$ shows the Sink and Tuttle model that the level of education influences the levels of productivity in the Research Unit and Social Organization (UPOS). The units (INPE, CBPF, CETEM) are those with higher level of technology. In the analysis of correlation was up eight variables that stood out and it became evident that the main construct of the model for UPOS was the efficiency. Regarding the most important variables were the two main work efficiency and quality, reinforcing the idea that the characteristics of plants influence the performance of the units. There is consensus among the units that the workforce is below the needs of each unit.
\end{abstract}

Keywords: Performance Evaluation; Sink and Tuttle model; MCT.

Data do recebimento do artigo: 29/07/2013

Data do aceite de publicação: 02/02/2014

Revista de Administração e Inovação, São Paulo, v. 11, n.2, p. 51-70, abr./jun. 2014. 